\title{
Antropologia e saúde pública no contexto de emergência sanitária global: uma perspectiva crítica latino-americana
}

\section{Anthropology and public health in the context of a global health emergency: a critical Latin American perspective}

\author{
Anaxsuell Fernando Silva ${ }^{1}$
}

1 - Universidade Federal da Integração Latino-Americana - UNILA, Foz do Iguaçu - PR, Brasil.

\section{RESUMO}

anaxsuell.silva@unila.edu.br

Palavras-chave:

Epidemiologia; Antropologia; Desigualdades.
Introdução: os aspectos socioculturais dos processos de saúde-doença têm sido, de maneira geral, considerados marginais ou irrelevantes para formulação das intervenções na área da saúde A crise sanitária causada pela Covid-19 impôs à comunidade de pesquisadores a busca de uma compreensão transversal de saúde. Objetivo: este artigo pretende problematizar a hegemonia biomédica no campo da saúde e como, no atual cenário, ela se desdobra em desigualdades em saúde. Método: para tanto, empreende uma revisão bibliográfica com ênfase no contexto latino-americano e explora fontes e argumentos em torno da necessidade de promover diálogo entre a Antropologia e a Epidemiologia. Resultados: a partir da bibliografia consultada, argumenta-se que a interconexão entre a Antropologia da saúde e a Epidemiologia Crítica Latino Americana se apresenta como caminho medular para a superação dos Colonialismos biomédicos, superação das desigualdades Sociais em Saúde e construção de uma soberania sanitária com forte conexão com a vida comunitária. Conclusão: a antropologia desenvolveu, ao longo das últimas décadas, significativo artefato teórico-metodológico para o estudo das formas de viver, enfermar-se e morrer. Tal repertório possibilita examinar as relações entre os modelos de compreensão no campo da saúde, a organização dos serviços, os programas de prevenção e as intervenções terapêuticas, além dos modelos culturais dos usuários. Por isso, não deve ser desprezada nas formulações das intervenções na área da saúde.
Keywords:

Epidemiology; Anthropo logy; Inequalities.

\begin{abstract}
Introduction: the sociocultural aspects of health-illness have generally been considered marginal or irrelevant to the formulation of interventions in the area. The health crisis caused by COVID-19 forced the research community to seek a transversal understanding of health. Objective: this article aims to discuss the biomedical hegemony in the field of health and how, in the current scenario, it unfolds in health inequalities. Method: to this end, it undertakes a literature review with an emphasis on the Latin American context and explores sources and arguments around the need to promote a dialogue between Anthropology and Epidemiology. Results: based on this bibliography, it is argued that the interconnection between Medical Anthropology and Latin American Critical Epidemiology presents itself as a key path to overcoming biomedical colonialisms, overcoming social inequalities in health and building a sanitary sovereignty with a strong connection with community life. Conclusion: anthropology has developed, over the last few decades, significant theoretical methodological approaches to the study of ways of living, getting sick and dying. This repertoire makes it possible to examine models for understanding health, the organization of services, prevention programs and therapeutic interventions, in addition to the users' cultural models. Therefore, it should not be neglected in the formulation of interventions in the health area.
\end{abstract}




\section{INTRODUÇÃO}

A crise sanitária do ano de 2020 trouxe à tona um conjunto transformações ao mundo em que vivemos. Tão rápidas, quanto profundas, estas mudanças socioculturais se espraiaram em diferentes dimensões da vida e suscitaram perplexidade ante sua velocidade e amplitude. Este cenário de emergência em saúde pública impôs esforços de reflexão no sentido de compreender o que se passa no mundo e intervir sobre a realidade.

Hegemônico atualmente, o modelo biomédico científico ocidental tem sua gênese associada ao contexto da Revolução Artístico-Cultural, transcorrida a partir do século XVI, no âmbito do Renascimento. René Descartes (1596-1650) definiu o seu "método para bem conduzir a razão e procurar a verdade nas ciências", 1 isto é, um conjunto de regras que constituem os fundamentos de construção do conhecimento científico no ocidente. De acordo com o método cartesiano, não pode ser aceito, como verdade, nada que não possa ser identificado como tal. Assim, dever-se-ia separar cada elemento ou aspecto do objeto em estudo e examinar em tantas partes quanto sejam possíveis e necessárias para se chegar a uma solução; cabe mencionar também a condução do pensamento de forma ordenada, partindo do simples ao mais complexo; e a necessidade de efetuar uma revisão exaustiva dos diversos componentes de um argumento. Este seu método é apresentado num livro denominado de Discurso do Método, publicado originalmente em 1637.

$\mathrm{Na}$ esteira deste método ocidental de apreensão do conhecimento, o saber biomédico conceitualizou doença como desajuste ou falha nos mecanismos de adaptação do organismo ou ainda ausência de reação aos estímulos a cuja ação está exposto, um processo que conduz a uma perturbação da estrutura ou da função de um órgão, de um sistema ou de todo o organismo ou de suas funções vitais. ${ }^{2} \mathrm{E}$ dedicou-se, progressivamente, ao longo da história da medicina, à explicação da doença. Como desdobramento, este saber biomédico estuda o corpo em partes cada vez menores, compartimentando os saberes e reduzindo a saúde a um funcionamento mecânico. ${ }^{3}$

Ao refletir sobre a constituição este modelo, Capra $^{4}$ criticou a formulação fragmentária do modelo biomédico e argumentou que este se manifesta semelhante a uma teoria mecanicista, na qual o indivíduo humano é visto como corpo-máquina; o médico, por sua vez, é atua como um mecânico; e a doença, seria o defeito da máquina. Esta percepção do indivíduo como uma máquina é demarcável historicamente e remonta ao advento do capitalismo.

Deste modo, é possível dizer que a intervenção de cuidado é fundada numa concepção que é simultaneamente reducionista e mecanicista. Para Foucault ${ }^{5}$ o cuidado também pode e deve ser compreendido como dispositivo de controle dos corpos circunscritos em um espaço social marcado por relações de poder, neste caso mereceria destaque o poder (bio)médico.

Os aspectos socioculturais têm sido, de maneira geral, considerados marginais ou irrelevantes para formulação das intervenções na área da saúde. Usualmente, considera-se apenas aquelas considerações relacionadas ao diagnóstico biomédico. Em direção contrária, desde os anos de 1970 os estudos demonstram a relevância de se pensar o processo saúde-doença desde uma perspectiva sociocultural. Uma vez que se trata de fenômenos complexos que conjugam fatores biológicos, sociológicos, econômicos, ambientais e culturais. A complexidade do objeto implica buscar uma compreensão transversal de saúde.

A antropologia desenvolveu, ao longo das últimas décadas, significativo artefato teóricometodológico para o estudo das formas de viver, enfermar-se e morrer. Tal repertório possibilita examinar as relações entre os modelos de compreensão no campo da saúde, a organização dos serviços, os programas de prevenção e as intervenções terapêuticas, além dos modelos culturais dos usuários.

$\mathrm{O}$ discurso antropológico tem apontado os limites da tecnologia biomédica e a insuficiência da abordagem hospitalocêntrica, farmacológica e laboratorial, quando se trata de mudar de forma o estado de saúde de uma população. As pesquisas etnográficas têm nos demonstrado que a saúde de uma população é desdobramento do seu modo de vida, do seu entorno social e cultural. Desta maneira, é possível apontar a importância de estabelecer uma relação de complementaridade entre os estudos epidemiológicos e aqueles oriundos das Ciências Sociais (antropológicos, sociológicos e psicológicos) no que diz respeito à saúde.

De acordo com Maurício Gomes Pereira ${ }^{6}$ a epidemiologia não é um tema novo, a disciplina acadêmica que utiliza este nome, sim é o que pode ser considerada recente. Trata-se de um conceito 
que engloba os fatores relacionados com a saúde da população.Estaárea do pensamento em saúde congrega métodos e técnicas de três campos do conhecimento: Estatística, Ciências da Saúde e Ciências Sociais para estudar o processo saúde-doença, como este fator é distribuído e seus determinantes (ou, como veremos ao longo deste texto, determinações) na sociedade. Em linhas gerais, a Epidemiologia se define como:

ciência que estuda o processo saúdedoença em coletividades humanas, analisando a distribuição e os fatores determinantes das enfermidades, danos à saúde e eventos associados à saúde coletiva, propondo medidas específicas de prevenção, controle ou erradicação de doenças, e fornecendo indicadores que sirvam de suporte ao planejamento, administração e avaliação das ações de saúde. ${ }^{7}$

Nos estudos epidemiológicos predominam os estudos quantitativos com interesse nas abordagens sobre os comportamentos dos indivíduos. A incidência ou prevalência de uma certa patologia e as características de indivíduos relacionados a ela são determinadas, no intuito de conhecer os perfis de distribuição da patologia em grupos ou fatores de risco associados. $\mathrm{Na}$ abordagem sociológica, os problemas de saúde são apreendidos em sua dimensão social e não individual. As Ciências Sociais da saúde investigam a determinação que exercem os contextos social e institucional sobre as enfermidades e os comportamentos delas decorrentes. A antropologia, por exemplo, considera que a saúde e tudo que se relaciona a ela são noções socialmente construídos e culturalmente interpretados. ${ }^{8}$ Neste sentido, a perspectiva etnográfica, ou dito de outro modo, a abordagem qualitativa, é empregada para identificar e analisar a mediação que exercem os fatores sociais e culturais na construção de formas características de pensar e agir frente à saúde e à doença. Integrando uma apreensão da dimensão cultural, a antropologia ao lado da epidemiologia, podem contribuir para ampliar o contexto que deve ser levado em consideração na leitura dos processos patológicos.

A despeito da evidente necessidade de complementaridade, estes dois campos de conhecimento atuam, geralmente, apartados e disjuntos devido a compartimentalização da produção de saberes em saúde. A profunda dicotomia entre métodos quantitativos e qualitativos ainda afeta o conjunto das ciências da saúde, obscurece a complementaridade dessas duas estratégias de pesquisa. Diversos autores ${ }^{9-11}$ sugerem que as perspectivas quantitativas e qualitativas deveriam ser encaradas como perspectivas complementares, como fases sequenciais de um mesmo processo.

É nesta direção que este trabalho caminha. Pretendemos a partir de uma revisão bibliográfica explorar fontes e argumentar em torno da necessidade de promover diálogo entre a Antropologia e a Epidemiologia, de maneira mais específica discutiremos as pontes entre a Antropologia da saúde e a Epidemiologia Crítica Latino Americana como caminhos medulares para a superação dos Colonialismos biomédicos e superação das desigualdades Sociais em Saúde.

\section{Desenvolvimento}

Em junho de 2019, o departamento de população da $\mathrm{ONU}^{12}$ divulgou um conjunto de novas projeções populacionais para sociedade. Naquela ocasião, a população mundial para foi estimada em 7,79 bilhões para o ano de 2020 e apontava que chegaríamos ao contingente de 8 bilhões nos próximos três anos. Estes indivíduos que habitam o planeta terra não são iguais. Do ponto de vista espacial, tal população está distribuída em espaços nacionais e continentais que apresentam diferentes configurações demográficas e geopolíticas. Além das notórias diferenças fenotípicas e culturais, identificase significativas diferenças socioeconômicas. As clivagens sociais decorrentes das desigualdades são produtos de processos históricos complexos. Algumas destas distinções que poderiam ser apenas diferenças, transmutaram-se em desigualdades devido a relações desiguais de poder as quais limitam o acesso e a posse aos bens, serviços e riqueza que são fruto do trabalho coletivo e acumulado através de gerações, são desigualmente distribuídos. ${ }^{13,14}$

Estas diferenças, elaboradas e convertidas em desigualdade, frequentemente deslocam-se para exprimir-se na saúde. Tornam-se perceptíveis e modulam desiguais condições de saúde a partir de aspectos raciais, de gênero, de classe e das suas intersecções. Expressam-se tanto em camadas de riscos à saúde, quanto no acesso diferenciado aos recursos disponíveis no sistema de saúde. ${ }^{15}$ Dito de outra maneira, as desigualdades observadas na saúde estão diretamente relacionadas às desigualdades 
observáveis em outras esferas da vida social. As desigualdades na saúde se materializam em discrepantes níveis de oportunidades de acesso aos avanços científicos e tecnológicos do campo da saúde da sociedade moderna, assim como distintas chances de adoecimento, modos de enfrentamento desta doença e morte. ${ }^{14,16,17}$ As desigualdades sociais em saúde estão presentes em todos os países independente do seu poderio econômico, da quantidade de riqueza acumulada. No contexto do capitalismo, desigualdade reafirma-se numa estrutura social perversa. Frequentemente tais desigualdades convertem-se em iniquidades em saúde.

Aqui, parece importante pontuar quais seriam as diferenças conceituais entre desigualdades e iniquidades em saúde. Enquanto a primeira diz respeito àquelas diferenças evidentes e mensuráveis presentes tanto nas condições de saúde, quanto relacionadas às diferenças no acesso aos serviços de prevenção, cura ou reabilitação da saúde (desigualdades no cuidado à saúde); Iniquidade em saúde, por sua vez, está relacionada às desigualdades decorrentes de alguma forma de injustiça. ${ }^{18}$

Em seus escritos, Margareth Whitehead ${ }^{19}$ estabeleceu um vínculo direto entre iniquidade $\mathrm{e}$ injustiça, a professora de Saúde Pública da University of Liverpool aponta que a iniquidade pode ser compreendida como uma desigualdade desnecessária e evitável sobre saúde. Na mesma direção, Escorel ${ }^{20}$ argumenta que o termo iniquidade tem uma dimensão política e ética, assim, toda desigualdade redutível é iniqua por ser produto dos conflitos em uma arena permeada por interesses contraditórios.

Nos últimos anos, a expansão da subjetividade neoliberal ${ }^{21,22}$ marcada pelo individualismo têm sustentado a percepção de que os eventos ocorridos na sociedade são de responsabilidade dos indivíduos neles implicados, rechaçando o caráter coletivo e social da vida. Esta perspectiva de mundo fundamenta a naturalização das desigualdades por parte da sociedade e faz com que os atores políticos as entendam como resultantes de problemas individuais, minimizando as expressões das injustiças e, por conseguinte, colaborando para escassez de políticas e ações governamentais para minimizá-las.

A epidemiologia e a antropologia da saúde são disciplinas científicas que buscam por padrões de doenças, comportamento e de modos de enfrentamento da enfermidade. Elas estão separadas por suas histórias e tradição disciplinar - a "Epidemiologia tende a ser mais estatística e quantitativa e a Antropologia textual e qualitativa" ${ }^{23}$ A. Neste trabalho, o principal argumento se dá em torno da defesa de um diálogo integrado entre ambas as disciplinas, na mesma direção de outros autores ${ }^{24,25,26,11,27,28}$

Esta relação de complementaridade entre epidemiologia e antropologia passou a fazer parte do escopo na saúde coletiva, levando em conta a perspectiva de integração metodológica nas investigações em saúde. Em um primeiro momento, as discussões enfatizaram as evidentes dualidades como quantitativo/qualitativo ou objetividade/ subjetividade, posicionando o método particular a cada uma das disciplinas em extremidades opostas por suas características.

O método epidemiológico, com a premissa de separação radical entre sujeito-objeto, engendrase historicamente sustentada por uma noção ideal de rigor científico, o qual poderia ser mensurado pela objetividade e a neutralidade das suas investigações. A epidemiologia trabalha com explicações causais formuladas a partir de pesquisas que se valem de instrumentos como entrevistas estruturadas. Com isso, se busca medir a abrangência e representatividade dos dados obtidos.

Por outro lado, o método antropológico desfaz o distanciamento entre sujeito-objeto. A pesquisa etnográfica é concebida a partir da intersubjetividade, do erro e do inesperado e estes aspectos se impõem a qualquer discurso em torno da objetividade. A ênfase está nas histórias de vida, nas relações entre os indivíduos e suas cosmovisões. Assim, por meio da observação participante a antropologia busca compreender e interpretar o significado das práticas sociais, considerando os distintos contextos culturais em que emergem.

Se antes havia disputas de competência a qual buscava definir o melhor método, estas podem ser substituídas por uma perspectiva complexa, inclusiva e de hibridização metodológica. Na medida em que admitirmos a possibilidade de integração de métodos, reconheceremos os limites de cada um deles e trabalhamos para conter as lacunas compreensivas que decorrem da existência de tais limitações.

Obviamente, a integração entre epidemiologia e antropologia é uma oportunidade que não está desprovida de tensões, contradições ou disputas de poder, as quais envolvem sobretudo a maneira como tais disciplinas estruturaram historicamente suas bases conceituais e premissas metodológicas. As 
experiências de pesquisas em saúde existentes que apontam para uma aproximação entre epidemiologia e antropologia ocorre ao mesmo tempo em que significativas transformações no discurso científico contemporâneo se manifestam, e que geralmente enfatizam, valores mais próximos ao olhar antropológico, caracterizado por abordar mais de perto seus sujeitos de pesquisa, isto é, considera a unidade de situações singulares.

Dito de outra maneira, aspectos como subjetividade, autonomia e diferença expressaram-se mais vigorosamente na saúde coletiva por volta de uma década atrás, incidindo nas perspectivas de pesquisa até então hegemônicas na área, as quais privilegiavam a concepção de ciência neutra e objetiva.

É importante dizer que não se trata de migrar de um extremo, onde se localiza a objetividade, para outro que seria a subjetividade. Ou transitar do universal para o singular, do quantitativo para o qualitativo. Tampouco operar a partir da dicotomia entre os critérios quantitativos e qualitativos. O desafio que se impõe é mover-se entre os diferentes níveis e modos de compreensão e apreensão da realidade

A integração metodológica pressupõe outra adversidade. As já explicitadas diferenças metodológicas existentes remetem às finalidades da pesquisa e também à construção de seus objetos. Nesta direção, não se advoga pela integração de dois objetos - o epidemiológico e o antropológico. A proposta de integração acredita que a formulação dos problemas de investigação pode ser concebida de maneira integrada. Para que tal ponte entre estes campos de conhecimento se efetive, é preciso que as disciplinas em questão empreendam um movimento autorreflexivo que pretenda repensar as bases nas quais elas estão fundadas. Este movimento tem dado origem a um modo específico de se fazer e pensar epidemiologia, gestado no continente latinoamericano.

Como já discutimos aqui, a epidemiologia convencional está tradicionalmente assentada no paradigma empírico-funcionalista, e busca traçar a relação entre a saúde e fatores (de risco e proteção) que muitas vezes não se reconectam como as discussões consolidadas no campo das Ciências Sociais. Assim, Jaime Breilh, epidemiológo equatoriano, tem se preocupado, basicamente em retomar a categoria da reprodução social, dos modos de viver, adoecer e morrer como mote analítico para superar os modelos epistêmicos lineares e causalistas que a epidemiologia convencional insiste em reafirmar. ${ }^{29,11}$

É nesta direção, portanto, que, em Breilh, a "determinação social em saúde" junto com as categorias de "reprodução social" e "metabolismo sociedade-natureza" compõem os três eixos a partir dos quais se estruturam o pensamento da epidemiologia crítica latino-americana que emerge na segunda metade do século $\mathrm{XX} .^{28}$

Ainda no decorrer dos anos de 1970, a América Latina vivenciou o desenvolvimento de um campo do saber chamado de Medicina Social. Ela se colocava como uma resposta a um modelo desenvolvimentista que vinha sendo implementado de maneira exaustiva nas políticas latino-americanas, e em consequência, produza efeitos marcantes no campo da saúde pública. ${ }^{30,31}$

A ideia que circulava no continente à época era que o crescimento econômico levaria a uma melhora automática das condições de vida e saúde, contudo, o que se averiguou foi o oposto. Ainda que os indicadores macroeconômicos ostentassem melhora, os sociais degringolavam. Um novo cenário se configurava: enquanto os gastos com saúde aumentavam e novas tecnologias floresciam, o acesso à saúde seguia cerceado e as condições gerais de saúde pioravam. ${ }^{27}$

Nesse cenário, a correlação entre condições de saúde e desigualdade social se explicitaram. Diversos autores, preocupados em compreender o que se enxergava, com abordagens teórico-metodológicas diversificada, passaram a produzir um conhecimento científico crítico e sensível às questões sociais, tal esforço seria reconhecido como Medicina Social.

Quem primeiro empregou o termo "Medicina Social" para designar modos de enfrentamento das formas de adoecimento salientando as condições sociais que alteram a fisiologia considerada normal foi Jules Guérin, ainda em 1848.

Em lugar das abordagens imprecisas e descoordenadas que costumamos incluir sob a denominação de polícia médica, saúde pública, medicina forense, chegou o momento de reunir todas elas em um todo organizado e elevá-las a seu mais alto nível sob o nome de Medicina Social, nome que melhor expressa seu propósito. $^{32}$

E, ao destacar a correspondência entre as condições de pauperização e insalubridade dos 
trabalhadores, na Inglaterra do século XIX, o próprio Guérin explicita o intento da Medicina Social fazendo uso de uma situação concreta:

Uma grande dúvida está presente hoje em dia em todos nós: quais seriam os efeitos dos salários mais altos e horas de trabalho mais curtas? Examinada à luz da Medicina Social, esta pergunta se responde fácil e satisfatoriamente. Horas de trabalho mais curtas significarão maior energia e maior bem-estar [...] que resultarão numa força de trabalho melhor e mais produtiva. ${ }^{32}$

Nunes $^{33}$ sublinhou que foi na década de 1840, em um contexto revolucionário, que muitos médicos, filósofos e pensadores das questões públicas assumiram o caráter social da medicina e da doença. O debate público a respeito da relação saúdesociedade ganhou pujança entre o final da década de 1960 e início da década de 1970. Havia uma enorme influência das discussões teóricas oriundas de autores das ciências humanas, notadamente da sociologia e da antropologia.

Neste sentido, muitos autores ${ }^{33}$ consideram $^{2}$ que a conferência de Michel Foucault, no Rio de Janeiro, em 1974, sobre as origens da Medicina Social foi um marco significativo. Paralelamente, nesse momento histórico, verifica-se em outras partes da América Latina uma produção teórica relevante, que assumia os vínculos existentes entre as práticas de saúde e a dinâmica social. Assim, os subsídios teóricos e analíticos das ciências sociais ao estudo da saúde foram indispensáveis para que se chegasse ao estágio atual de sistematização dos conhecimentos no campo.

Ao discutir a proposta de medicina social, Sérgio Arouca afirma:

A Medicina Social aparece, pois, com duas tendências; a primeira [...] movimento de modificação da medicina ligado à própria mudança de sociedade, ou [...] através de sua mudança institucional [...]; a segunda é uma tentativa de redefinir a posição e o lugar dos objetos dentro da medicina, de fazer demarcações conceituais, colocar em questão quadros teóricos, enfim, trata-se de um movimento ao nível da produção de conhecimentos que, reformulando as indagações básicas que possibilitaram a emergência daMedicinaPreventiva, tenta definir um objeto de estudo nas relações entre o biológico e o psicossocial. A medicina social, elegendo como campo de investigação estas relações, tenta estabelecer uma ciência que se situa nos limites das ciências atuais. ${ }^{34}$

Neste ponto percebemos a distinção das duas dimensões propositivas da Medicina Social: de um lado, a formulação de propostas de intervenção na vida social e na medicina baseadas na conexão saúdesociedade e, por outro, a proposta de consolidar uma ramificação de estudos dessa conexão, que se expresse tanto no aspecto do adoecimento, quanto na reflexão a respeito da produção da assistência médica e das práticas profissionais nos serviços. Obviamente, está contida uma crítica a determinado modelo de medicina - cara, fragmentada e com parcos resultados para a saúde da população.

Entrava em crise o modelo desenvolvimentista de saúde pública e a sua postulação causal entre os efeitos do crescimento econômico e melhora das condições de saúde. ${ }^{35}$ Isso era especialmente apropriado para compreender o Brasil deste período, que, a despeito de vivenciar um momento de crescimento econômico, não percebia resultados concretos refletidos nas condições de vida de sua população.

Sob efeito destas ideias, o movimento latinoamericano da epidemiologia crítica, questionaria a capacidade explicativa da epidemiologia convencional, clássica, a respeito da realidade, que, a despeito de dispor de um arsenal estatístico robusto, não cumpriria com a tarefa de relacionar os sistemas sociais, modos de vida e saúde, e tampouco é efetivo na explicação a respeito da distribuição "por classes das formas e intensidades de exposição a situações de risco, ou os diferentes níveis de vulnerabilidade inerentes a cada realidade social distinta". ${ }^{28}$

Além das questões conceituais, a epidemiologia crítica latino-americana sintetiza os problemas metodológicos que impossibilitam que análises críticas e aprofundadas sejam feitas seguindo moldes de uma epidemiologia convencional:

a) uma explicação fenomenológica reducionista e fragmentária da realidade e da saúde, b) primazia absoluta de causa e efeito como grande organizador e lógica do universo epidemiológico, 
c) reprodução da relação causa-efeito como um artefato formal aplicado para identificar fatores de risco, d) a redução da noção de exposição/vulnerabilidade a um problema probabilístico essencialmente individual, e e) redução da organização da prática epidemiológica em ações funcionais sobre fatores de risco. ${ }^{29}$

O modelo clássico, convencional, da epidemiologia fundamenta-se em paradigmas hegemônicos do pensamento ocidental. É efeito do colonialismo médico. Nesta perspectiva quaisquer processos serão explicados apenas no plano dos fenômenos observáveis empiricamente, a realidade é tratada como algo que existe "em si". A saúde ou doença, que emerge empiricamente, são as únicas coisas comprováveis, e, portanto, passíveis de existência. Por isso, as avaliações quantitativas são consideradas meios de obtenção de conhecimento que "deduzem as relações simples e lineares que se estabelecem entre cada fenômeno". ${ }^{28}$

A epidemiologia crítica latino-americana surge, portanto, como resposta a uma epidemiologia ortodoxa moderna, hegemônica e colonial que, constrói-se sobre fundamentos tecnicistas, abstratos e alheios à vida concreta das pessoas. Por isso consideramos que o desenvolvimento de uma mudança epistemológica no interior da epidemiologia poderá ser o ponto de articulação para se compreender as reflexões antropológicas a respeito da saúde. Esse repertório crítico combinado, dialogado, é um passo paradigmático importante para que a saúde caminhe na trilha do pensamento crítico e siga pensando sobre seus próprios (des)acertos e comprometa-se com a eliminação das desigualdades.

Em síntese, a intenção deste texto tem sido demonstrar através desta revisão teórico-bibliográfica que a epidemiologia clássica empreende suas análises sustentadas em noções de pessoa, tempo e lugar descoladas do conhecimento antropológico e por isso obsoletas; somemos a esta consideração que também aos conceitos de história natural da doença e sistemas de equilíbrio (antígeno-suscetível-ambiente) falham na tarefa de conhecer a realidade para alterá-la. ${ }^{29,11}$

\section{CONCLUSÃO}

Ainda que a discussão a respeito das possibilidades de diálogo e integração entre a epidemiologia e a antropologia já tenha em seu repertório uma significativa literatura - alguns, inclusive, chegaram a cunhar o termo "epidemiologia comportamental" (Fernandes, 2003) nos anos 70 este trabalho não é uma revisão sistemática a respeito das contribuições já existentes no que tange à relação da epidemiologia com a Antropologia ou com outras disciplinas. ${ }^{36,37}$ Em outra direção, o objetivo foi explorar os aspectos teórico-metodológicos, a partir de alguns autores que têm permitido a reflexão sobre as potencialidades de uma abordagem que integre as referidas disciplinas para o estudo dos fenômenos de saúde. Dentre as abordagens possíveis, optou-se por revisar o que tem sido sistematizado em torno de uma epidemiologia crítica latino-americana.

A compreensão do diálogo se dá matizando o pensamento epidemiológico como cerne de uma perspectiva crítica radical à sociedade capitalista e a suas formas de dominação, que se desdobram em desigualdades sociais expressas em desigualdades em saúde. Tal cenário não pode ser compreendido à luz de um colonialismo médico, ou a partir da aceitação tácita das interpretações das questões relacionadas a saúde oriundas de uma perspectiva biomédica, hospitalocêntrica e centrada em fármacos.

Ao longo deste trabalho tentamos problematizar a necessidade de se romper com a falsa dicotomia entre os métodos qualitativos e quantitativos. E, para fazê-lo, seria necessário localizar o objeto de estudo - as pessoas - no núcleo central do problema. E isso só pode ser feito a partir da construção de ponte, de um canal de diálogo entre a Antropologia da saúde e a Epidemiologia Crítica Latino Americana. Este é caminhos medulares para a superação dos Colonialismos biomédicos e superação das desigualdades Sociais em Saúde.

\section{REFERÊNCIAS}

1. Pinto H. A Medicina no "Discurso do Método" de Descartes: Um Breve Apontamento. Arquivos de Medicina 2009;23(1):236.

2. Herzlich C. Saúde e doença no início do século XXI: entre a experiência privada e a esfera pública. Physis: Revista de Saúde Coletiva 2004;14(2):383-94.

3. Barros JA. Pensando o processo saúde doença: a que responde o modelo biomédico? Saúde e Sociedade 2002;11(1):67-84.

4. Capra F. O ponto de mutação. São Paulo: Cultura 1982.

5. Foucault M. Microfísica do poder. Rio de Janeiro: Edições Graal 1979.

6. Pereira MG. Epidemiologia: teoria e prática. Rio de Janeiro: Ed. Guanabara 2002. 
7. Rouquayrol MZ, Almeida Filho N. Epidemiologia \& Saúde. Rio de Janeiro: MEDSI 2003.

8. Nichter M. Anthropology and International Health: South Asian Case Studies. Dordrechet: Kluwer Publications 1989.

9. Minayo MC, Sanches O. Quantitativo-qualitativo: oposição ou complementaridade? Cad Saúde Púb 1993;9:239-62.

10. Soares CB. Os desafios da epidemiologia crítica e as respostas do capitalismo às suas dificuldades de reprodução: o caso da esfera cibernética. Revista Brasileira de Epidemiologia 2015;18(4):990-4. https://doi.org/10.1590/19805497201500040028

11. Breilh J. Critical Epidemiology and the People's Health. New York: Oxford University Press 2021.

12. United Nations. O Perspectivas Mundiais de População 2019. Disponível em: https://population.un.org/wpp/.

13. Stiglitz JE. The price of inequality. How today's divide society endangers our future. New York: WW Norton \& Company 2013.

14. Barreto ML. Desigualdades em Saúde: uma perspectiva global. Ciênc Saúde Colet 2017;22(7). https://doi. org/10.1590/1413-81232017227.02742017

15. Marmot M. The health gap: the challenge of an unequal world. Bloomsbury, London 2015.

16. Michelin C. Determinação social do processo saúde-doença: que vozes ecoam no Brasil? (Mestrado em Saúde Pública). Rio de Janeiro: Escola Nacional de Saúde Pública Sergio Arouca, Fundação Oswaldo Cruz 2015.

17. López-Arellano O. Determinación social de la salud: desafios y agendas posibles. Divulgação em Saúde para Debate 2013: 150-156.

18. Kawachi I, Subramanian S, Almeida-Filho N. A glossary for health inequalities. Journal of Epidemiology Community Health 2002, 56 (9): 647-652.

19. Whitehead M. The concepts and principles of equity and health. International Journal of Health Services 1992; 22:42945.

20. Escorel S. Equidade em saúde. In: Pereira IB, Lima JCF. Dicionário da educação profissional em saúde. 2. ed. Rio de Janeiro: Escola Politécnica em Saúde Joaquim Venâncio, 2008. 21. Dardot P, Laval C. A Nova Razão do Mundo: Ensaio sobre a sociedade neoliberal. São Paulo: Boitempo, 2016.

22. Safatle V, Silva Junior N, Dunker C. (Orgs) Neoliberalismo como gestão do sofrimento psíquico. Belo Horizonte: Autêntica, 2020 .

23. Trostle, JA. Epidemiologia e Cultura. Rio de Janeiro: Fiocruz 2013.

24. Hahn RA. Sickness and Healing: An Anthropological Perspective. New Haven, Yale University Press 1995.

25. Dressler WW, Bindon JR. The Health Consequences of Cultural Consonance: Cultural Dimensions of Lifestyle, Social Support, and Arterial Blood Pressure in an African American Community. American Anthropologist 2000;102(2):244-60.

26. Breilh, J. Epidemiologia, Economia, Medicina y Política. Mexico: Fontamara 1998.
27. Iriart C. Medicina social latinoamericana: aportes y desafíos. Rev Panam Salud Publica 2002;12(2):128-36.

28. Souza IFP, Mendes Á, Carnut L. História política e pensamento epidemiológico: Breilh e a economia política da saúde. Revista Guillermo de Ockham 2019;17(1):77-84. https:// doi.org/10.21500/22563202.4039

29. Breilh, J. La determinación social de la salud como herramienta de transformación hacia una nueva salud pública. Revista Facultad Nacional de Salud Pública 2013, 31 (1): 13-27. 30. Nunes ED. Saúde coletiva: história de uma ideia e de um conceito. Saúde e Sociedade 1994;3(2):5-21.

31. Laurell AC. La política social y de salud en América Latina: un campo de lucha política. Cad. Saúde Pública 2017;33(supll2). https://doi.org/10.1590/0102-311X000439162017

32. Garcia JC. Entrevista com Garcia. Em E. D. Nunes, As ciências sociais em saúde na America Latina: tendências e perspectivas 1985 Brasília, DF: OPS. 21-28.

33. Nunes ED. Saúde coletiva: história recente, passado antigo. Em C. G. (Org.), Tratado de saúde coletiva. Rio de Janeiro: Fiocruz 2007.

34. Arouca AS. O dilema preventivista: contribuição para a compreensão e crítica da medicina preventiva. São Paulo: Fio Cruz 2003.

35. Nunes ED. Saúde coletiva: história de uma ideia. Saúde e Sociedade 1994;3(2):5-21.

36. Janes CSR. Anthropology, and epidemiology: interdisciplinary aproaches to the study of health and disease. Dordrecht: Reidel Publishing Company 1986.

37. Sallis JFON. Behavioral epidemiology: a systematic framework to classify phases of research on health promotion and disease prevention. Annals of Behavioral Medicine 2000;22(4):294-8.

Recebido em: 14/12/2020

Aceito em:15/04/2021

Como citar: SILVA, Anaxsuell Fernando. Antropologia e saúde pública no contexto de emergência sanitária global: uma perspectiva crítica latino-americana. Revista Interdisciplinar de Promoção da Saúde, Santa Cruz do Sul, v. 3, n. 4, out 2020. ISSN 2595-3664. Disponível em: <https://online.unisc.br/seer/index.php/ripsunisc/article/view/16085>. Acesso em: 01 out. 2020. doi:https://doi.org/10.17058/rips.v3i4.16085 